\title{
Selective alkylation of aminophenols
}

\author{
Renchao Wang and Jiaxi $\mathrm{Xu}^{*}$
}

State Key Laboratory of Chemical Resource Engineering, and Department of Organic Chemistry, Faculty of Science, Beijing University of Chemical Technology, Beijing 100 029, China

E-mail:jxxu@mail.buct.edu.cn

\begin{abstract}
O- or N-Alkylated derivatives of aminophenols are important synthetic intermediates in organic synthesis. A series of aminophenols were selectively alkylated on their hydroxyl group in good yields via benzaldehyde protection of the amino group, subsequent alkylation, and hydrolysis; or on their amino group via imination and following reduction.
\end{abstract}

Keywords: Alkylation, alkyl halide, aminophenol, alkoxyaniline, selectivity

\section{Introduction}

O- or N-Alkylated derivatives of aminophenols (alkoxyanilines and N-alkylaminophenols) are important synthetic intermediates in organic synthesis. They have been widely used in the synthesis of pharmaceutical agents, ${ }^{1-4}$ such as non-peptide full agonists for the human bradykinin $\mathrm{B}_{2}$ receptor $^{1}$ and cholesterol acyltransferase inhibitors, ${ }^{2}$ and chiral ligands for asymmetric catalysis., ${ }^{5,6}$ Both of alkoxyanilines and $\mathrm{N}$-alkylaminophenols can be prepared from aminophenols via alkylation. However, a mixture of O- and N-alkylated, and N,O-dialkylated derivatives of aminophenols generated when aminophenols were alkylated directly with alkyl halides without good selectivity in most cases. It is a tedious work to separate and purify them. Thus, the development of new synthetic strategies which allow access to O- or N-alkylated derivatives of aminophenols selectively is of interest to both laboratory and industry preparation of alkoxyanilines and $\mathrm{N}$-alkylaminophenols.

O-Alkylated derivatives of aminophenols were synthesized via the reduction of nitroalkoxybenzenes, ${ }^{7}$ or via the amino protection and hydroxyl alkylation of aminophenols, and subsequent deprotection strategy, in which the amino groups were protected generally with acetyl and phthalyl which required harsh removing conditions, ${ }^{8,9}$ or expensive tertbutoxycarbonyl (Boc) and benzoxycarbonyl (Cbz) ${ }^{10,11}$ To synthesize chiral ligands for the asymmetric catalysis, we hoped to prepare o-benzyloxyanilines as starting materials. We designed and applied an efficient and inexpensive strategy, in which inexpensive benzaldehyde 
was used to protect the amino group of aminophenols. When we extended the route to synthesize various alkylated derivatives of aminophenols, we found that Morel and co-workers reported a similar method for the synthesis of $o$-allyloxyaniline via the condensation of $o$-aminophenol and benzaldehyde in dichloromethane under the catalysis of alumina oxide for $24 \mathrm{~h}$, the allylation of the resulting N-benzylideneaminophenol with allyl bromide for $36 \mathrm{~h}$ using potassium carbonate as a base, and subsequent hydrolysis in $1998 .{ }^{12}$ Only one example was described in their report. Compared to their method, our procedure is more efficient and practical. Herein, we report our procedure for selective synthesis of $\mathrm{O}$ - and $\mathrm{N}$-alkylated derivatives of aminophenols from aminophenols.

\section{Results and Discussion}

To search an efficient procedure to prepare alkoxyanilines from various commercially available aminophenols, we selected the imination of $o$-aminophenol with benzaldehyde as a model reaction to optimize the reaction conditions. $o$-Aminophenol and benzaldehyde were condensed in methanol directly to give rise to $N$-benzylideneaminophenol in an excellent yield of $96.4 \%$ without any catalyst. Thus, we paid much attention to the alkylation step. It is necessary more than 2 days for complete benzylation of $o$-aminophenol at RT. However, we found that the rate of benzylation could be speeded up by increasing reaction temperature. The excellent yield was obtained under refluxing for $20 \mathrm{~h}$ with equimolar benzyl bromide. To add more amount of benzyl bromide did not further improve the yield, adversely, resulting in the formation of further $\mathrm{N}$ benzylated derivative during hydrolysis in the next step. Although the treatment with saturated sodium bisulfite can hydrolyze the imine group of the product and remove benzaldehyde conveniently, the hydrolysis occurred incompletely. The benzylated product can also be hydrolyzed with silica gel when it was purified on a silica gel column to afford the desired product benzyloxyaniline in a satisfactory yield (74.5\%). It has been found that it is the best method to hydrolyze it with hydrochloride solution to afford benzyloxyaniline in the yield of 93.5\% after simple washing with dichloromethane and neutralization. A series of alkoxyanilines were prepared under optimal reaction conditions (Scheme 1). The results are summarized in Table 1.

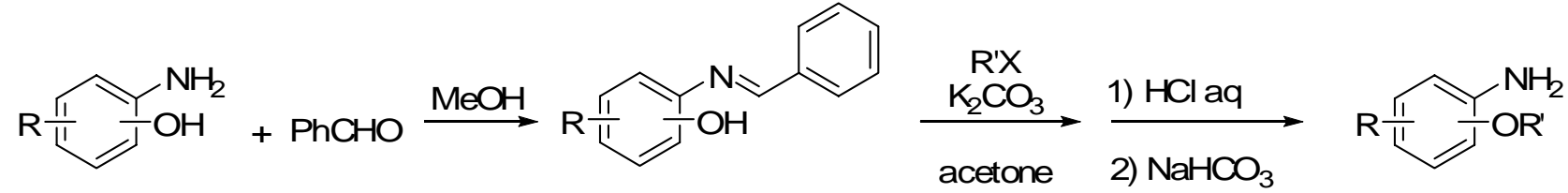

Scheme 1. Selective alkylation of the hydroxyl group of aminophenols.

More active alkyl halides, such as benzyl and allyl bromides, produced the corresponding 
alkoxyanilines in good to excellent yields (Table 1, entries 1 and 2). Primary alkyl halides, such as methyl iodide, $n$-pentyl bromide, even long chain dodecyl bromide, gave rise to the alkoxyanilines in satisfactory yields (Table 1, entries 3-5). However, secondary alkyl halide isopropyl bromide and sec-butyl iodide yielded the desired products in lower yields with recovered starting materials (Table 1 , entries 6 and 7). For substituted $o$-aminophenols and $p$ aminophenols, satisfactory to good yields were obtained in the selective alkylation (Table 1 , entries 8-11).

Table 1. Selective alkylation of hydroxyl group of aminophenols

\begin{tabular}{|c|c|c|c|c|c|}
\hline Entry & Aminophenol & Yield (\%) & $\mathrm{R}^{\prime} \mathrm{X}$ & Product & Yield (\%) \\
\hline 1 & & & $\mathrm{BnBr}$ & & 93.5 \\
\hline 2 & & & AllBr & & 82.2 \\
\hline 3 & & & MeI & & 53.8 \\
\hline 4 & & 96.4 & $n-\mathrm{C}_{5} \mathrm{H}_{11} \mathrm{Br}$ & & 62.8 \\
\hline 5 & & & $n-\mathrm{C}_{12} \mathrm{H}_{25} \mathrm{Br}$ & & 67.4 \\
\hline 6 & & & $i-\mathrm{PrBr}$ & & 38.7 \\
\hline 7 & & & $s$-BuI & & 31.5 \\
\hline 8 & & 95.2 & & & 86.1 \\
\hline 9 & & 94.2 & & & 57.2 \\
\hline 10 & $\mathrm{OH}$ & 97.9 & & & 63.0 \\
\hline 11 & $\mathrm{HC}$ & 88.9 & & & 76.4 \\
\hline
\end{tabular}


We also prepare the $\mathrm{N}$-alkylated derivatives of aminophenols selectively via the condensation of aminophenols and an aldehyde and subsequent reduction with sodium borohydride in a one-pot reaction (Scheme 2). ${ }^{13-15}$ The excellent overall yields were achieved for all given examples. The results are listed in Table 2.

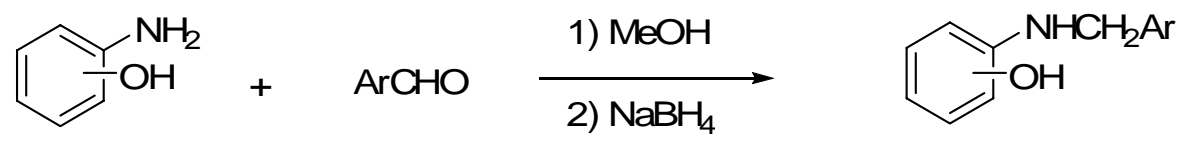

Scheme 2. Selective alkylation of the amino group of aminophenols.

Table 2. Selective alkylation of the amino group of aminophenols

Entry Aminophenol

In summary, aminophenols were selectively alkylated on their hydroxyl group via condensation with benzaldehyde, alkylation with alkyl halides, and hydrolysis with hydrochloride; and on their amino group with condensation with aldehydes and subsequent reduction with sodium borohydride in a one-pot reaction. In most cases, good to excellent yields were achieved. The current strategy is an economic and practical route for selective alkylation of aminophenols. 


\section{Experimental Section}

General. Melting points were obtained on a Yanaco-M500 melting point apparatus. The ${ }^{1} \mathrm{H}$ NMR spectra were recorded on a Varian Mercury 200 or Varian Mercury Plus 300 spectrometer with TMS as an internal standard in the $\mathrm{CDCl}_{3}$ solution. TLC separations were performed on silica gel GF-254 plates with petroleum ether $\left(60-90{ }^{\circ} \mathrm{C}\right)$ /ethyl acetate $(10: 1)$; the plates were visualized with UV light.

\section{Selective alkylation of the hydroxyl group of aminophenols. General procedures}

To a stirred solution of aminophenol (30 mmol) in $80 \mathrm{~mL}$ of methanol was added benzaldehyde (3.18 g, $30 \mathrm{mmol}$ ) and the resulting solution was stirred for $1 \mathrm{~h}$. After removal of the solvent in vacuo, the residue was recrystallized from ethanol to afford phenylmethyleneaminophenol as grey crystals.

To a stirred solution of phenylmethyleneaminophenol $(3 \mathrm{mmol})$ in acetone $(30 \mathrm{ml})$ was added $\mathrm{K}_{2} \mathrm{CO}_{3}$ (828 mg, $6 \mathrm{mmol}$ ) and alkyl halide ( $3 \mathrm{mmol}$ ) and the mixture was refluxed for $20 \mathrm{~h}$. After cooling, the inorganic precipitate was filtered off through a Celite pad and the filtrate was concentrated in vacuo. To the resulting residue was added $10 \mathrm{ml}$ of dichloromethane and $30 \mathrm{ml}$ of $1 \mathrm{~N} \mathrm{HCl}$ and the mixture was vigorously stirred for $1 \mathrm{~h}$. After separated with a separatory funnel, the aqueous layer was neutralized with $\mathrm{NaHCO}_{3}$, then extracted with dichloromethane. The combined organic phase was dried over $\mathrm{NaSO}_{4}$, and the solvent was removed in vacuo to afford the alkyloxyaniline as colorless or yellowish oil, or crystals.

2-Benzyloxyaniline. Colorless crystals; mp 38-40 ${ }^{\circ} \mathrm{C}$, Lit. ${ }^{10} 37-38{ }^{\circ} \mathrm{C},{ }^{1} \mathrm{H} \mathrm{NMR}(300 \mathrm{MHz}$, $\left.\mathrm{CDCl}_{3}\right) \delta: 3.83$ (s, 2H, $\mathrm{NH}_{2}$ ), 5.08 (s, 2H, $\mathrm{CH}_{2}$ ), 6.68-6.88 (m, 4H, ArH), 7.33-7.46 (m, 5H, ArH). 2-(Allyloxy)aniline. ${ }^{12}$ Yellowish oil; ${ }^{1} \mathrm{H}$ NMR (300 MHz, $\left.\mathrm{CDCl}_{3}\right) \delta$ : 3.82 (s, 2H, $\mathrm{NH}_{2}$ ), 4.56 (dt, $J=5.4,1.5 \mathrm{~Hz}, 2 \mathrm{H}, \mathrm{CH}_{2}$ ), 5.28 (dq, $J=10.5,1.5 \mathrm{~Hz}, 1 \mathrm{H},=\mathrm{CH}_{2}$ ), 5.41 (dq, $J=17.4,1.5 \mathrm{~Hz}, 1 \mathrm{H}$, $=\mathrm{CH}_{2}$ ), 6.08 (ddt, $\left.J=10.5,17.4,5.4 \mathrm{~Hz}, 1 \mathrm{H},=\mathrm{CH}\right), 6.68-6.83(\mathrm{~m}, 4 \mathrm{H}, \mathrm{ArH})$.

2-Methoxyaniline. ${ }^{16}$ Yellowish oil; ${ }^{1} \mathrm{H}$ NMR (300 MHz, $\left.\mathrm{CDCl}_{3}\right) \delta$ : $3.78\left(\mathrm{~s}, 2 \mathrm{H}, \mathrm{NH}_{2}\right.$ ), 3.85 (s, $\left.3 \mathrm{H}, \mathrm{CH}_{3}\right), 6.71-6.81$ (m, 4H, ArH).

2-Pentyloxyaniline. ${ }^{17}$ Yellowish oil; ${ }^{1} \mathrm{H}$ NMR (300 $\left.\mathrm{MHz}, \mathrm{CDCl}_{3}\right) \delta: 0.93(\mathrm{t}, J=7.2 \mathrm{~Hz}, 3 \mathrm{H}$, $\mathrm{CH}_{3}$ ), 1.32-1.51 (m, 4H, $\mathrm{CH}_{2}$ ), 1.77-1.86 (m, 2H, $\mathrm{CH}_{2}$ ), $3.80\left(\mathrm{~s}, 2 \mathrm{H}, \mathrm{NH}_{2}\right), 3.98$ (t, $J=6.6 \mathrm{~Hz}$, $\left.2 \mathrm{H}, \mathrm{CH}_{2}\right), 6.68-6.81$ (m, 4H, ArH).

2-Dodecyloxyaniline. Yellowish crystals; mp 36-37 ${ }^{\circ} \mathrm{C}$, Lit. ${ }^{18}$ 37-38 ${ }^{\circ} \mathrm{C},{ }^{1} \mathrm{H}$ NMR (300 MHz, $\left.\mathrm{CDCl}_{3}\right) \delta: 0.88\left(\mathrm{t}, J=6.9 \mathrm{~Hz}, 3 \mathrm{H}, \mathrm{CH}_{3}\right), 1.26\left(\mathrm{~s}, 16 \mathrm{H}, \mathrm{CH}_{2}\right), 1.44-1.46\left(\mathrm{~m}, 2 \mathrm{H}, \mathrm{CH}_{2}\right), 1.76-1.85$ (m, 2H, $\mathrm{CH}_{2}$ ), 3.93 (s, 2H, $\mathrm{NH}_{2}$ ), 3.98 (t, $\left.J=6.6 \mathrm{~Hz}, 2 \mathrm{H}, \mathrm{CH}_{2}\right), 6.70-6.80$ (m, 4H, ArH).

2-Isopropoxyaniline. Colorless crystals; mp 139-141 ${ }^{\circ} \mathrm{C}$, Lit. ${ }^{19} 137-139{ }^{\circ} \mathrm{C},{ }^{1} \mathrm{H}$ NMR $(300 \mathrm{MHz}$, $\left.\mathrm{CDCl}_{3}\right) \delta: 1.35$ (d, $\left.J=6.3 \mathrm{~Hz}, 6 \mathrm{H}, \mathrm{CH}_{3}\right), 3.79$ (s, 2H, $\mathrm{NH}_{2}$ ), 4.53 (m, 1H, CH), 6.67-6.82 (m, 4H, $\mathrm{ArH})$.

2-sec-Butoxyaniline. ${ }^{20}$ Colorless crystals; mp 151-153 ${ }^{\circ} \mathrm{C},{ }^{1} \mathrm{H}$ NMR $\left(200 \mathrm{MHz}, \mathrm{CDCl}_{3}\right) \delta: 0.99$ (t, $J=7.4 \mathrm{~Hz}, 3 \mathrm{H}, \mathrm{CH}_{3}$ ), 1.31 (d, $J=6.2 \mathrm{~Hz}, 3 \mathrm{H}, \mathrm{CH}_{3}$ ), 1.57-1.85 (m, 2H, $\mathrm{CH}_{2}$ ), 3.81 (br, 2H, $\mathrm{NH}_{2}$ ), 4.23-4.38 (m, 1H, CH), 6.67-6.81 (m, 4H, ArH). 
5-Methyl-2-benzyloxyaniline. ${ }^{21}$ Colorless crystals; mp 32-33 ${ }^{\circ} \mathrm{C},{ }^{1} \mathrm{H}$ NMR $\left(300 \mathrm{MHz}, \mathrm{CDCl}_{3}\right) \delta$ : 2.22 (s, 3H, $\mathrm{CH}_{3}$ ), 3.78 (s, 2H, NH N, 5.05 (s, 2H, $\mathrm{CH}_{2}$ ), 6.50 (dd, $J=1.8,8.1 \mathrm{~Hz}, 1 \mathrm{H}, \operatorname{ArH}$ ), 6.58 (d, $J=1.8 \mathrm{~Hz}, 1 \mathrm{H}, \mathrm{ArH}), 6.75$ (d, $J=8.1 \mathrm{~Hz}, 1 \mathrm{H}, \mathrm{ArH}), 7.32-7.45$ (m, 5H, ArH).

5-Chloro-2-benzyloxyaniline. Yellowish crystals; mp 53-55 ${ }^{\circ} \mathrm{C}$, Lit. $^{22} 60{ }^{\circ} \mathrm{C},{ }^{1} \mathrm{H}$ NMR $(200$ $\mathrm{MHz}_{\mathrm{CDCl}}$ ) $\delta: 3.89$ (s, 2H, $\mathrm{NH}_{2}$ ), 5.04 (s, 2H, $\mathrm{CH}_{2}$ ), 6.60-6.75 (m, 3H, ArH), 7.35-7.39 (m, 5H, ArH).

5-Nitro-2-benzyloxyaniline. Yellowish crystals; mp 106-108 ${ }^{\circ} \mathrm{C}$, Lit. $^{23} 104.5-105.5{ }^{\circ} \mathrm{C}$, ${ }^{1} \mathrm{H}$ NMR (300 MHz, $\left.\mathrm{CDCl}_{3}\right) \delta: 4.12$ (s, 2H, NH ), 5.17 (s, 2H, $\mathrm{CH}_{2}$ ), 6.85 (d, J = 9.0 Hz, 1H, ArH), 7.36-7.43 (m, 5H, ArH), 7.56 (d, $J=2.7 \mathrm{~Hz}, 1 \mathrm{H}, \mathrm{ArH}), 7.63$ (dd, $J=2.7,8.7 \mathrm{~Hz}, 1 \mathrm{H}, \mathrm{ArH}$ ).

4-Benzyloxyaniline. Colorless crystals; mp 39-40 ${ }^{\circ} \mathrm{C}$, Lit. ${ }^{24} 44-45{ }^{\circ} \mathrm{C}$, ${ }^{1} \mathrm{H}$ NMR (300 MHz, $\left.\mathrm{CDCl}_{3}\right) \delta: 3.43\left(\mathrm{~s}, 2 \mathrm{H}, \mathrm{NH}_{2}\right.$ ), $4.98\left(\mathrm{~s}, 2 \mathrm{H}, \mathrm{CH}_{2}\right.$ ), 6.64 (d, $\left.J=9.0 \mathrm{~Hz}, 2 \mathrm{H}, \mathrm{ArH}\right), 6.81$ (d, $J=9.0$ $\mathrm{Hz}, 2 \mathrm{H}, \mathrm{ArH}), 7.30-7.44$ (m, 5H, ArH).

\section{Selective alkylation of the amino group of aminophenols. General procedures}

To a stirred solution of aminophenol (3 mmol) in $20 \mathrm{~mL}$ of methanol was added aldehyde (3 $\mathrm{mmol}$ ) and the solution was stirred for $1 \mathrm{~h}$. After cooling, sodium borohydride (216 mg, $6 \mathrm{mmol}$ ) was then added portionwise. After stirred for another $1 \mathrm{~h}$, it was poured into $30 \mathrm{~mL}$ of water. After extracted with dichloromethane, the combined organic phase was dried over $\mathrm{NaSO}_{4}$, and the solvent was removed in vacuo to obtain alkylaminophenol as colorless or yellowish crystals. 2-(Benzylamino)phenol. Colorless crystals; mp 68-69 ${ }^{\circ} \mathrm{C}$, Lit. ${ }^{25}$ 69-71 ${ }^{\circ} \mathrm{C},{ }^{1} \mathrm{H} \mathrm{NMR}(300 \mathrm{MHz}$, $\left.\mathrm{CDCl}_{3}\right) \delta: 4.33$ (s, 2H, $\mathrm{CH}_{2}$ ), 4.65 (br, 1H, NH), 6.61-6.82 (m, 4H, ArH), 7.24-7.39 (m, 5H, ArH). 2-(2-Hydroxyphenylmethyl)aminophenol. Yellowish crystals; mp 94-96 ${ }^{\circ} \mathrm{C}$, Lit. ${ }^{14}$ 99-101 ${ }^{\circ} \mathrm{C}$ ${ }^{1} \mathrm{H}$ NMR (300 MHz, $\mathrm{CDCl}_{3}$ ) $\delta: 4.38$ (s, 2H, $\mathrm{CH}_{2}$ ), 6.01 (br, 2H, NH and $\left.\mathrm{OH}\right), 6.72$ (m, 2H, ArH), 6.83-6.87 (m, 4H, ArH), 7.13-7.23 (m, 2H, ArH).

2-(4-Methoxyphenylmethyl)aminophenol. Colorless crystals; mp 121-122 ${ }^{\circ} \mathrm{C}$, Lit. ${ }^{26} 121{ }^{\circ} \mathrm{C},{ }^{1} \mathrm{H}$ NMR (200 MHz, $\left.\mathrm{CDCl}_{3}\right) \delta: 3.80$ (s, 3H, $\left.\mathrm{CH}_{3}\right), 3.81$ (br, 2H, NH and $\left.\mathrm{OH}\right), 4.26\left(\mathrm{~s}, 2 \mathrm{H}, \mathrm{CH}_{2}\right.$ ), 6.65-6.69 (m, 2H, ArH), 6.85-6.90 (m, 4H, ArH), 7.28-7.32 (m, 2H, ArH).

2-(4-Chlorophenylmethyl)aminophenol. Yellowish crystals; mp 104-105 ${ }^{\circ} \mathrm{C}$, Lit. ${ }^{13} 108-109{ }^{\circ} \mathrm{C}$, ${ }^{1} \mathrm{H}$ NMR (300 MHz, $\mathrm{CDCl}_{3}$ ) $\delta: 4.30$ (s, 2H, $\mathrm{CH}_{2}$ ), 4.66 (br, 2H, NH and $\left.\mathrm{OH}\right), 6.57-6.83$ (m, 4H, ArH), 7.24-7.31 (m, 4H, ArH).

4-(Benzylamino)phenol. Colorless crystals; mp 97-98 ${ }^{\circ} \mathrm{C}$, Lit. ${ }^{27}$ 91-93 ${ }^{\circ} \mathrm{C},{ }^{1} \mathrm{H}$ NMR $(300 \mathrm{MHz}$, $\left.\mathrm{CDCl}_{3}\right) \delta: 4.05$ (br, 2H, NH and $\left.\mathrm{OH}\right), 4.26\left(\mathrm{~s}, 2 \mathrm{H}, \mathrm{CH}_{2}\right), 6.55$ (d, $\left.J=8.7 \mathrm{~Hz}, 2 \mathrm{H}, \mathrm{ArH}\right), 6.68$ (d, $J$ $=8.7 \mathrm{~Hz}, 2 \mathrm{H}, \mathrm{ArH})$, 7.27-7.36 (m, 5H, ArH).

\section{Acknowledgements}

This work was supported by the National Natural Science Foundation of China (Project No. 20772005). 


\section{References}

1. Sawada, Y.; Kayakiri, H.; Abe, Y.; Mizutani, T.; Inamura, N.; Asano, M.; Hatori, C.; Aramori, I.; Oku, T.; Tanaka, H. J. Med. Chem. 2004, 47, 2853.

2. Asano, S.; Ban, H.; Kino, K.; Ioriya, K.; Muraoka. M. Bioorg. Med. Chem. 2009, 17, 4636.

3. Ford, R. E.; Knowles, P.; Lunt, E.; Marshall, S. M.; Penrose, A. J.; Ramsden, C. A.; Summers, A. J. H.; Walker, J. L.; Wright, D. E. J. Med. Chem. 1986, 29, 538.

4. Mayer, S.; Guillaumet, G.; Merour, J. Y. Heterocycles 2001, 55, 1873.

5. Mashiko, T.; Hara, K.; Tanaka, D.; Fujiwara, Y.; Kumagai, N.; Shibasaki, M. J. Am. Chem. Soc. 2007, 129, 11342.

6. Mashiko, T.; Kumagai, K.; Shibasaki, M. Org. Lett. 2008, 10, 2725.

7. Dobson, D.; Todd, A.; Gilmore, J. Synth. Commun. 1991, 21, 611.

8. Ek, A.; Witkop, B. J. Am. Chem. Soc. 1954, 76, 5579.

9. Humm, A. W.; Schneider, M. R. Arch. Pharm. 1990, 323, 83.

10. Dubash, N. P.; Mangu, N. K.; Satyam, A. Synth. Commun. 2004, 34, 1791.

11. Sajiki, H.; Hirota, K. Tetrahedron 1998, 54, 13981.

12. Morel, G.; Marchanda, E.; Benjelloun, A. T. Sinbandhit, S.; Guillouc, O.; Gall, P. Eur. J. Org. Chem. 1998, 2631.

13. Varma, R. S.; Dahiya, R. Tetrahedron 1998, 54, 6293.

14. Itoh, T.;Nagata, K.; Miyazaki, M.;Ishikawa, H.; Kurihara, A.; Ohsawa, A. Tetrahedron 2004, $60,6649$.

15. Balsells, J.; Carroll, P. J.; Walsh, P. J. Inorg. Chem. 2001, 40, 5568.

16. Vo, G. D.; Hartwig, J. F. J. Am. Chem. Soc. 2009, 131, 11049.

17. Nodzu, R.; Watanabe, H.; Kuwata, S.; Nagaishi, C.; Shibuya, K.; Teramatsu, T.; Arima, H.; Kobayashi, K.; Maizuru, H. Yakugaku Zasshi 1952, 72, 543 (Chem. Abstr. 1953, 47, 15772).

18. Kunitz, F. W.; Pelz, W.; Nittel, F. BE 729233, 1969 (Chem. Abstr. 1970, 73, 4972).

19. Downer-Riley, N. K.; Jackson, Y. A. Tetrahedron 2007, 63, 10276.

20. Bermudez, J.; Dabbs, S.; King, F. D. J. Med. Chem. 1990, 33, 1932.

21. Keller, E. GB 711232, 1954 (Chem. Abstr. 1954, 48, 81197).

22. (Chemische Fabriek L. van der Grinten N. V.) NL 964461960 (Chem. Abstr. 1960, 58, 21115).

23. Verkade, P. E.; Roelfzema, H.; Meerburg, W.; Bosma, G. S. K. Recl. Trav. Chim. Pays-Bas. 1947, 66, 374.

24. Takasaki, M.; Motoyama, Y.; Higashi, K. Org. Lett. 2008, 10, 1601.

25. Endo, Y.; Shudo, K.; Okamoto, T. Synthesis 1983, 471.

26. Samet, A. V.; Kislyi, K. A.; Marshalkin, V. N.; Semenov, V. V. Russ. Chem. Bull. 2006, 55, 549.

27. Shafir, A.; Buchwald, S. L. J. Am. Chem. Soc. 2006, 128, 8742. 